EPJ Web of Conferences 112,01018 (2016)

DOI: $10.1051 /$ epjconf/201611201018

(C) Owned by the authors, published by EDP Sciences, 2016

\title{
Probing the transversity spin structure of a nucleon in neutrino- production of a charmed meson
}

\author{
B. Pire ${ }^{1, a}$, L. Szymanowski ${ }^{2, b}$, and J. Wagner ${ }^{2, c}$ \\ ${ }^{1}$ Centre de physique théorique, École Polytechnique, CNRS, Université Paris-Saclay, 91128 Palaiseau, \\ France \\ ${ }^{2}$ National Centre for Nuclear Research (NCBJ), Warsaw, Poland
}

\begin{abstract}
Including $O\left(m_{c}\right)$ terms in the coefficient functions and/or $O\left(m_{D}\right)$ twist 3 contributions in the heavy meson distribution amplitudes leads to a non-zero transverse amplitude for exclusive neutrino production of a $D$ pseudoscalar charmed meson on an unpolarized target. We work in the framework of the collinear QCD approach where chiral-odd transversity generalized parton distributions (GPDs) factorize from perturbatively calculable coefficient functions.
\end{abstract}

\section{Introduction}

The now well established framework of collinear QCD factorization [1-3] for exclusive reactions mediated by a highly virtual photon in the generalized Bjorken regime describes hadronic amplitudes using generalized parton distributions (GPDs) which give access to a 3-dimensional analysis [4] of the internal structure of hadrons. Neutrino production is another way to access (generalized) parton distributions [5]. Although neutrino induced cross sections are orders of magnitudes smaller than those for electroproduction and neutrino beams are much more difficult to handle than charged lepton beams, they have been very important to scrutinize the flavor content of the nucleon and the advent of new generations of neutrino experiments opens new possibilities. In particular, the flavor changing character of the electroweak current allows charmed quark to be produced in processes involving light quark partonic distributions [6]. This in turn allows helicity flip hard amplitudes to occur at the $O\left(\frac{m_{c}}{Q}\right)$ level where $Q$ is the typical large scale allowing QCD collinear factorization. Such a coefficient function has to be attached to a chiral-odd generalized parton distribution, the elusive transversity GPDs [7-9]. The transverse character of these GPDs select the transverse polarization of the $W$-boson, which phenomenologically allows a separation of this interesting amplitude through the azimuthal distribution of the final state particles [6].

\footnotetext{
a e-mail: bernard.pire@polytechnique.edu

be-mail: lech.szymanowski@ncbj.gov.pl

ce-mail: jakub.wagner@ncbj.gov.pl
} 


\section{Kinematics}

For definiteness, we consider the exclusive production of a pseudoscalar $D$-meson through the reaction (see Fig. 1):

$$
v_{l}(k) N\left(p_{1}\right) \rightarrow l^{-}\left(k^{\prime}\right) D^{+}\left(p_{D}\right) N\left(p_{2}\right),
$$

where $\mathrm{N}$ is a proton or a neutron, in the kinematical domain where collinear factorization leads to a description of the scattering amplitude in terms of nucleon GPDs and the $D$-meson distribution amplitude, with the hard subprocesses:

$$
W^{+}(\varepsilon, q) d \rightarrow D^{+}\left(p_{D}\right) d .
$$

Our kinematical notations are as follows ( $m$ and $M_{D}$ are the nucleon and $D$-meson masses, $m_{c}$ will denote the charmed quark mass):

$$
\begin{aligned}
& q=k-k^{\prime} \quad ; \quad Q^{2}=-q^{2} \quad ; \quad \Delta=p_{2}-p_{1} \quad ; \quad \Delta^{2}=t ; \\
& p_{1}^{\mu}=(1+\xi) p^{\mu}+\frac{1}{2} \frac{m^{2}-\Delta_{T}^{2} / 4}{1+\xi} n^{\mu}-\frac{\Delta_{T}^{\mu}}{2} ; \quad p_{2}^{\mu}=(1-\xi) p^{\mu}+\frac{1}{2} \frac{m^{2}-\Delta_{T}^{2} / 4}{1-\xi} n^{\mu}+\frac{\Delta_{T}^{\mu}}{2} \\
& q^{\mu}=-2 \xi^{\prime} p^{\mu}+\frac{Q^{2}}{4 \xi^{\prime}} n^{\mu} ; p_{D}^{\mu}=2\left(\xi-\xi^{\prime}\right) p^{\mu}+\frac{M_{D}^{2}-\Delta_{T}^{2}}{4\left(\xi-\xi^{\prime}\right)} n^{\mu}-\Delta_{T}^{\mu},
\end{aligned}
$$

with $p^{2}=n^{2}=0$ and $p . n=1$. As in the double deeply virtual Compton scattering case [10], it is meaningful to introduce two distinct momentum fractions:

$$
\xi=-\frac{\left(p_{2}-p_{1}\right) \cdot n}{2} \quad, \quad \xi^{\prime}=-\frac{q \cdot n}{2} .
$$

Neglecting the nucleon mass and $\Delta_{T}$, the approximate values of $\xi$ and $\xi^{\prime}$ are

$$
\xi \approx \frac{Q^{2}+M_{D}^{2}}{4 p_{1} \cdot q-Q^{2}-M_{D}^{2}} \quad, \quad \xi^{\prime} \approx \frac{Q^{2}}{4 p_{1} \cdot q-Q^{2}-M_{D}^{2}} .
$$

To unify the description of the scaling amplitude, we define a modified Bjorken variable $x_{B}^{D} \equiv \frac{Q^{2}+M_{D}^{2}}{2 p_{1} \cdot q}$ which allows to express $\xi$ and $\xi^{\prime}$ in a compact form:

$$
\xi \approx \frac{x_{B}^{D}}{2-x_{B}^{D}} \quad, \quad \xi^{\prime} \approx \frac{x_{B}}{2-x_{B}^{D}} .
$$

If the meson mass is the relevant large scale (for instance in the limiting case where $Q^{2}$ vanishes as in the timelike Compton scattering kinematics [11]) :

$$
Q^{2} \rightarrow 0 ; \xi^{\prime} \rightarrow 0 ; \xi \approx \frac{\tau}{2-\tau} ; \tau=\frac{M_{D}^{2}}{s_{W N}-m^{2}}
$$

\section{The transverse amplitude}

In the Feynman gauge, the non-vanishing $m_{c}$-dependent part of the Dirac trace in the hard scattering part depicted in Fig. 1a reads:

$$
\operatorname{Tr}\left[\sigma^{p i} \gamma^{v} \hat{p}_{D} \gamma^{5} \gamma^{v^{\prime}} \frac{m_{c}}{D_{1}} \hat{\varepsilon}\left(1-\gamma^{5}\right) \frac{-g_{v v^{\prime}}}{D_{2}}\right]=\frac{2\left(Q^{2}+M_{D}^{2}\right)}{\xi} \varepsilon_{\mu}\left[\epsilon^{\mu p i n}+i g_{\perp}^{\mu i}\right] \frac{m_{c}}{D_{1}} \frac{1}{D_{2}}
$$




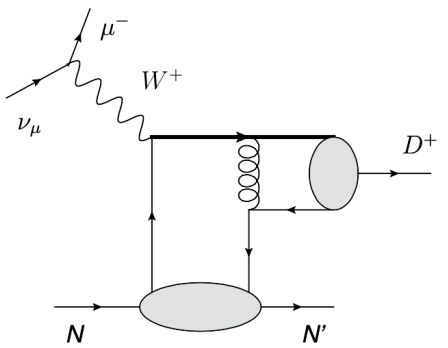

(a)

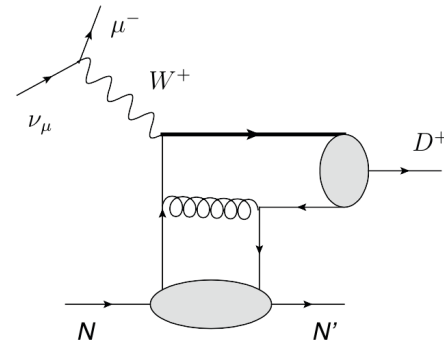

(b)

Figure 1. Feynman diagrams for the factorized amplitude for the $v_{\mu} N \rightarrow \mu^{-} D^{+} N^{\prime}$ or the $v_{\mu} N \rightarrow \mu^{-} D^{0} N^{\prime}$ process involving the quark GPDs; the thick line represents the heavy quark. In the Feynman gauge, diagram (a) involves convolution with both the transversity GPDs and the chiral even ones, whereas diagram (b) involves only chiral even GPDs.

where $\varepsilon$ is the polarization vector of the $W^{ \pm}$boson (we denote $\hat{p}=p_{\mu} \gamma^{\mu}$ for any vector $p$ ). The fermionic trace vanishes for the diagram shown on Fig. $1 \mathrm{~b}$ thanks to the identity $\gamma^{\rho} \sigma^{\alpha \beta} \gamma_{\rho}=0$. The denominators of the propagators read:

$$
\begin{aligned}
& D_{1}=k_{c}^{2}-m_{c}^{2}+i \epsilon=\frac{Q^{2}}{2 \xi^{\prime}}\left(x+\xi-2 \xi^{\prime}\right)-m_{c}^{2}+i \epsilon=\frac{Q^{2}+M_{D}^{2}}{2 \xi}(x+\xi)-Q^{2}-m_{c}^{2}+i \epsilon, \\
& D_{2}=k_{g}^{2}+i \epsilon=\bar{z}\left[\bar{z} m_{D}^{2}+\frac{Q^{2}+m_{D}^{2}}{2 \xi}(x-\xi)+i \epsilon\right],
\end{aligned}
$$

where $k_{c}\left(k_{g}\right)$ is the heavy quark (gluon) momentum. The transverse amplitude is then written as $(\tau=1-i 2)$ :

$$
T_{T}=\frac{i C \xi\left(m_{c}-2 M_{D}\right)}{\sqrt{2}\left(Q^{2}+M_{D}^{2}\right)} \bar{N}\left(p_{2}\right)\left[\mathcal{H}_{T}^{\phi} i \sigma^{n \tau}+\tilde{\mathcal{H}}_{T}^{\phi} \frac{\Delta^{\tau}}{m_{N}^{2}}+\mathcal{E}_{T}^{\phi} \frac{\hat{n} \Delta^{\tau}+2 \xi \gamma^{\tau}}{2 m_{N}}-\tilde{\mathcal{E}}_{T}^{\phi} \frac{\gamma^{\tau}}{m_{N}}\right] N\left(p_{1}\right),
$$

with $C=\frac{2 \pi}{3} C_{F} \alpha_{s} V_{d c}$, in terms of transverse form factors that we define as :

$$
\mathcal{F}_{T}^{\phi}=f_{D} \int \frac{\phi(z) d z}{\bar{z}} \int \frac{F_{T}^{d}(x, \xi, t) d x}{(x-\xi+\beta \xi+i \epsilon)(x-\xi+\alpha \bar{z}+i \epsilon)},
$$

where $F_{T}^{d}$ is any d-quark transversity GPD, $\alpha=\frac{2 \xi M_{D}^{2}}{Q^{2}+M_{D}^{2}}, \beta=\frac{2\left(M_{D}^{2}-m_{c}^{2}\right)}{Q^{2}+M_{D}^{2}}$ and we shall denote $\overline{\mathcal{E}}_{T}^{\phi}=$ $\xi \mathcal{E}_{T}^{\phi}-\tilde{\mathcal{E}}_{T}^{\phi}$. In the following, we shall put $\beta$ to 0 .

The prefactor in Eq.(10) shows the two sources of the transverse amplitude : $m_{c}$ signals the contribution from the helicity changing part of the heavy quark propagator, while $M_{D}$ signals the contribution from the twist 3 heavy meson distribution amplitude which we parametrize (omitting the Wilson 
lines) as :

$$
\left\langle 0\left|\bar{c}(y) \gamma^{5} d(-y)\right| D^{-}\left(P_{D}\right)\right\rangle=-i \frac{f_{D}}{4} M_{D} \int_{0}^{1} d z e^{i(2 z-1) P_{D} \cdot y} \phi_{D}^{(s)}(z),
$$

and for simplicity we identify $\phi^{s}$ with the leading twist 2 pseudoscalar charmed meson DA $\phi_{D}$ defined as:

$$
\left\langle 0\left|\bar{c}(y) \gamma^{\mu} \gamma^{5} d(-y)\right| D^{-}\left(P_{D}\right)\right\rangle=-i \frac{f_{D}}{4} P_{D}^{\mu} \int_{0}^{1} d z e^{i(2 z-1) P_{D} \cdot y} \phi_{D}(z) .
$$

\section{The azimuthal dependence of neutrinoproduction}

The dependence of a leptoproduction cross section on azimuthal angles is a widely used way to analyze the scattering mechanism. This procedure is helpful as soon as one can define an angle $\varphi$ between a leptonic and a hadronic plane, as for deeply virtual Compton scattering [12] and related processes. In the neutrino case, it reads :

$$
\begin{aligned}
& \frac{d^{4} \sigma\left(v N \rightarrow l^{-} N^{\prime} D\right)}{d x_{B} d Q^{2} d t d \varphi}= \\
& \tilde{\Gamma}\left\{\frac{1+\sqrt{1-\varepsilon^{2}}}{2} \sigma_{--}+\varepsilon \sigma_{00}+\sqrt{\varepsilon}(\sqrt{1+\varepsilon}+\sqrt{1-\varepsilon})\left(\cos \varphi \operatorname{Re} \sigma_{-0}+\sin \varphi \operatorname{Im} \sigma_{-0}\right)\right\}
\end{aligned}
$$

with

$$
\tilde{\Gamma}=\frac{G_{F}^{2}}{(2 \pi)^{4}} \frac{1}{16 x_{B}} \frac{1}{\sqrt{1+4 x_{B}^{2} m_{N}^{2} / Q^{2}}} \frac{1}{\left(s-m_{N}^{2}\right)^{2}} \frac{Q^{2}}{1-\epsilon},
$$

and the "cross-sections" $\sigma_{l m}=\epsilon_{l}^{* \mu} W_{\mu \nu} \epsilon_{m}^{v}$ are product of amplitudes for the process $W\left(\epsilon_{l}\right) N \rightarrow D N^{\prime}$, averaged (summed) over the initial (final) hadron polarizations. In the anti-neutrino case, one gets a similar expression with $\sigma_{--} \rightarrow \sigma_{++}, \sigma_{-0} \rightarrow \sigma_{+0}, 1+\sqrt{1-\varepsilon^{2}} \rightarrow 1-\sqrt{1-\varepsilon^{2}}$ and $\sqrt{1+\varepsilon}+$ $\sqrt{1-\varepsilon} \rightarrow \sqrt{1+\varepsilon}-\sqrt{1-\varepsilon}$. We use the standard notations of deep exclusive leptoproduction, namely $y=p_{1} . q / p_{1} \cdot k$ and $\epsilon \simeq 2(1-y) /\left[1+(1-y)^{2}\right]$. The azimuthal angle $\varphi$ is defined in the initial nucleon rest frame as:

$$
\sin \varphi=\frac{\vec{q} \cdot\left[\left(\vec{q} \times \vec{p}_{D}\right) \times(\vec{q} \times \vec{k})\right]}{|\vec{q}|\left|\vec{q} \times \vec{p}_{D} \| \vec{q} \times \vec{k}\right|},
$$

while the final nucleon momentum lies in the $x z$ plane $\left(\Delta^{y}=0\right)$.

The quantity $\sigma_{-0}$ is directly related to the observables $\langle\cos \varphi>$ and $\langle\sin \varphi>$ through

$$
\begin{aligned}
& \left\langle\cos \varphi>=\frac{\int \cos \varphi d \varphi d^{4} \sigma}{\int d \varphi d^{4} \sigma}=\frac{\sqrt{\varepsilon}(\sqrt{1+\varepsilon}+\sqrt{1-\varepsilon}) \mathcal{R} e \sigma_{-0}}{2 \epsilon \sigma_{00}+\left(1+\sqrt{1-\varepsilon^{2}}\right) \sigma_{--}}\right. \\
& \left\langle\sin \varphi>=\frac{\int \sin \varphi d \varphi d^{4} \sigma}{\int d \varphi d^{4} \sigma}=\frac{\sqrt{\varepsilon}(\sqrt{1+\varepsilon}+\sqrt{1-\varepsilon}) I_{m \sigma_{-0}}}{2 \epsilon \sigma_{00}+\left(1+\sqrt{1-\varepsilon^{2}}\right) \sigma_{--}} .\right.
\end{aligned}
$$

Estimating the counting rates and the angular observables defined above is in progress [13]. As a first step, we calculate $\sigma_{--}$which is bilinear in transversity quark GPDs. At zeroth order in $\Delta_{T}, \sigma_{--}$ reads:

$$
\sigma_{--}=\frac{4 \xi^{2} C^{2}\left(m_{c}+2 M_{D}\right)^{2}}{\left(Q^{2}+M_{D}^{2}\right)^{2}}\left\{\left(1-\xi^{2}\right)\left|\mathcal{H}_{T}^{\phi}\right|^{2}+\frac{\xi^{2}}{1-\xi^{2}}\left|\overline{\mathcal{E}}_{T}^{\phi}\right|^{2}-2 \xi \mathcal{R} e\left[\mathcal{H}_{T}^{\phi} \overline{\mathcal{E}}_{T}^{\phi *}\right]\right\} .
$$




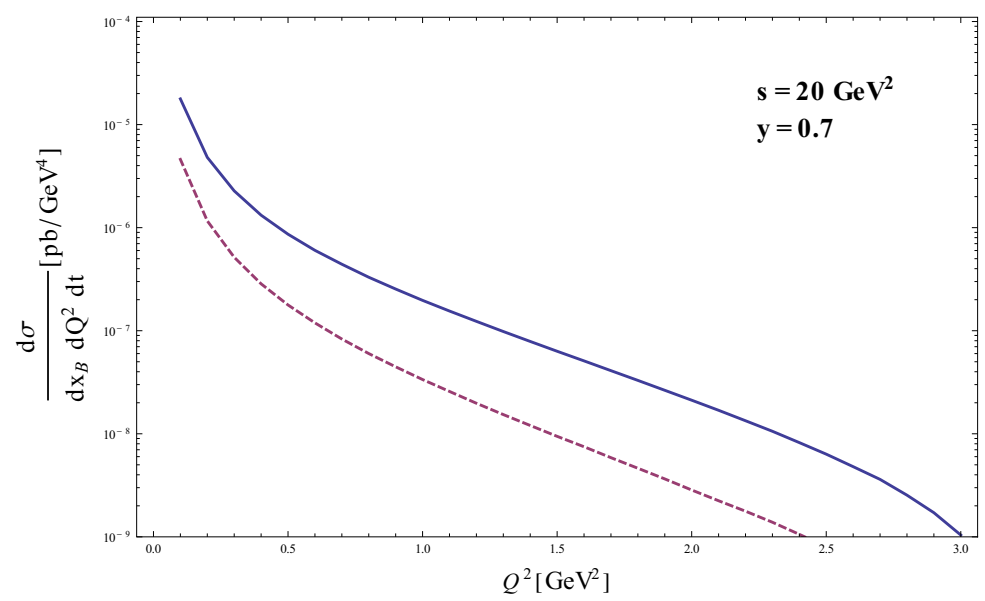

Figure 2. Contribution of $\sigma_{--}$to the differential cross section $\frac{d \sigma}{d x_{B} d Q^{2} d t}$ of neutrino-production of a $D^{+}$meson on a proton (dashed line) or a neutron (solid line) at $s=20 \mathrm{GeV}^{2}, y=0.7$ and $t=t_{0}$, as a function of $Q^{2}$.

Using the model of Ref [14] for the $D^{+}$meson distribution amplitude and the parametrization of the dominant transversity GPD $H_{T}(x, \xi, t)$ from Ref [15] (and neglecting for the time being other chiral-odd GPDs contributions), we compute the contribution to the differential cross section given in Eq.(13) integrated over $\varphi$. The result is shown in Fig. 2 as a function of $Q^{2}$ for $s=20 \mathrm{GeV}^{2}, y=0.7$ and $t=t_{\min }$. Since the process selects the $d$ - quark contribution, the proton and neutron target cases allow to access $H_{T}^{d}$ and $H_{T}^{u}$ respectively. Although small, the cross-sections are of the same order of magnitude as those for the neutrino production of $\pi$ or $D_{s}$ mesons estimated in [5]. This shows that these processes should be measurable in intense neutrino beam facilities.

Let us remind the reader that we allow $Q^{2}$ to be quite small since the hard scale governing our process is $M_{D}^{2}+Q^{2}$.

\section{Conclusion}

Collinear QCD factorization has allowed us to calculate neutrino production of $D$-mesons in terms of GPDs. Gluon and both chiral-odd and chiral-even quark GPDs contribute to the amplitude for different polarization states of the $W^{ \pm}$boson. The azimuthal dependence of the cross section allows to separate different contributions. Planned high energy neutrino facilities [16] which have their scientific program oriented toward the understanding of neutrino oscillations or elusive inert neutrinos may thus allow - without much additional equipment - some important progress in the realm of hadronic physics.

This work was partially supported by the COPIN-IN2P3 Agreement and by the french grant ANR PARTONS (Grant No. ANR-12-MONU-0008-01); L. Sz was partially supported by grant of National Science Center, Poland, No. 2015/17/B/ST2/01838.

\section{References}

[1] D. Müller et al., Fortsch. Phys. 42, 101 (1994). 
[2] X. Ji, Phys. Rev. D55, 7114 (1997); A. V. Radyushkin, Phys. Rev. D 56, 5524 (1997).

[3] J. C. Collins, L. Frankfurt, M. Strikman, Phys. Rev. D 56, 2982 (1997).

[4] M. Burkardt, Phys. Rev. D 62, 071503 (2000) [Phys. Rev. D 66, 119903 (2002)]; J. P. Ralston and B. Pire, Phys. Rev. D 66, 111501 (2002); M. Diehl and P. Hagler, Eur. Phys. J. C 44, 87 (2005).

[5] B. Lehmann-Dronke and A. Schafer, Phys. Lett. B 521 (2001) 55; C. Coriano and M. Guzzi, Phys. Rev. D 71 (2005) 053002; P. Amore, C. Coriano and M. Guzzi, JHEP 0502 (2005) 038; A. Psaker, W. Melnitchouk and A. V. Radyushkin, Phys. Rev. D 75 (2007) 054001; B. Z. Kopeliovich, I. Schmidt and M. Siddikov, Phys. Rev. D 86, 113018 (2012) and D 89, 053001 (2014); G. R. Goldstein, O. G. Hernandez, S. Liuti and T. McAskill, AIP Conf. Proc. 1222 (2010) 248.

[6] B. Pire and L. Szymanowski, Phys. Rev. Lett. 115, 092001 (2015); B. Pire and L. Szymanowski, arXiv: 1510.01869.

[7] M. Diehl, Eur. Phys. J. C 19, 485 (2001).

[8] M. Diehl et. al. Phys. Rev. D 59, 034023 (1999); J. C. Collins et. al., Phys. Rev. D 61, 114015 (2000).

[9] D. Yu. Ivanov et al., Phys. Lett. B 550, 65 (2002); R. Enberg, B. Pire and L. Szymanowski, Eur. Phys. J. C 47, 87 (2006).

[10] M. Guidal and M. Vanderhaeghen, Phys. Rev. Lett. 90, 012001 (2003); A. V. Belitsky and D. Mueller, Phys. Rev. Lett. 90, 022001 (2003).

[11] E. R. Berger, M. Diehl and B. Pire, Eur. Phys. J. C 23 (2002) 675.

[12] M. Diehl et al., Phys. Lett. B 411 (1997) 193; A. V. Belitsky, D. Mueller and A. Kirchner, Nucl. Phys. B 629, 323 (2002).

[13] B. Pire, L. Szymanowski and J. Wagner, work in progress.

[14] T. Kurimoto, H. n. Li and A. I. Sanda, Phys. Rev. D 65, 014007 (2002); T. Kurimoto, H. n. Li and A. I. Sanda, Phys. Rev. D 67, 054028 (2003).

[15] M. El Beiyad et. al., Phys. Lett. B 688, 154 (2010).

[16] D. S. Ayres et al. [NOvA Collaboration], hep-ex/0503053; J. Mousseau et al. [MINERvA Collaboration], arXiv:1601.06313 [hep-ex]; see also M. L. Mangano, S. I. Alekhin, M. Anselmino et al., CERN Yellow Report CERN-2004-002, pp.185-257 [hep-ph/0105155]. 